\title{
The effect of stellar evolution on the light curves' shapes of RR Lyrae stars in M3
}

\author{
Johanna Jurcsik \\ Konkoly Observatory of the Hungarian Academy of Sciences, P.O. Box \\ 67, $\mathrm{H}$-1525 Budapest
}

\begin{abstract}
The light curve characteristics of a homogeneous sample of variables in M3 are studied in detail. Accurate light curves and mean magnitudes of about 100 RRab and 50 RRc stars which do not show any type of modulation are analyzed. According to their mean magnitudes and Fourier parameters the variables can be sorted into four groups, representing different stages of the horizontal branch stellar evolution.
\end{abstract}

\section{Data}

The available CCD observations of $\mathrm{M} 3$ variables, as summarized in Table 1, enabled us to construct complete, accurate light curves (Fourier parameters and mean magnitudes) of a large number of variables. In Fig. 1 the light curves of variables in a very crowded and an outer regions are shown in order to demonstrate the different accuracies of the data. Though the differences in the scatter of the light curves are evident, it is not an easy task to estimate the accuracy of the mean magnitudes of the individual variables in the different data sets.

Table 1. CCD observations of M3 variables

\begin{tabular}{llcc}
\hline Reference & telescope & FOV & No. of var. \\
\hline Benkő et al. (2003, BJ03) & 1-m RCC Piszkástető & $5^{\prime} \times 5^{\prime}$ & 184 \\
& 60/90-cm Schmidt & $21^{\prime} \times 28^{\prime}$ & 225 \\
Corwin \& Carney (2001, CC01) & 0.9-m Kitt Peak & $23^{\prime} \times 23^{\prime}$ & 207 \\
Kaluzny et al. (1998, K98) & 1-m JKT La Palma & $2 \times 6.7^{\prime} \times 6.7^{\prime}$ & 42 \\
Carretta et al. (1998, C98) & 1.52-m RC Loiano $2 \times 2.40^{\prime} \times 4.16^{\prime}$ & 60 \\
\hline \hline
\end{tabular}

The BJ03 and CC01 observations covered the whole cluster providing light curves for nearly all of the variables. In contrast, K98 and C98 measured only selected, not central areas of the cluster. All the observations with the exception of BJ03 were reduced using standard crowded field point-spread function (PSF) photometry packages which could not provide accurate light curves and mean magnitudes of the most crowded variables. The BJ03 observations were extracted using the image subtraction method (ISIS, Alard 2000) which yielded light curves of significantly smaller scatter than any PSF photometry could provide (for a comparison see, e.g., Olech et al. 1999). The zero point of the ISIS method relies on the PSF photometry of the best images (with the best seeing), 
V 84

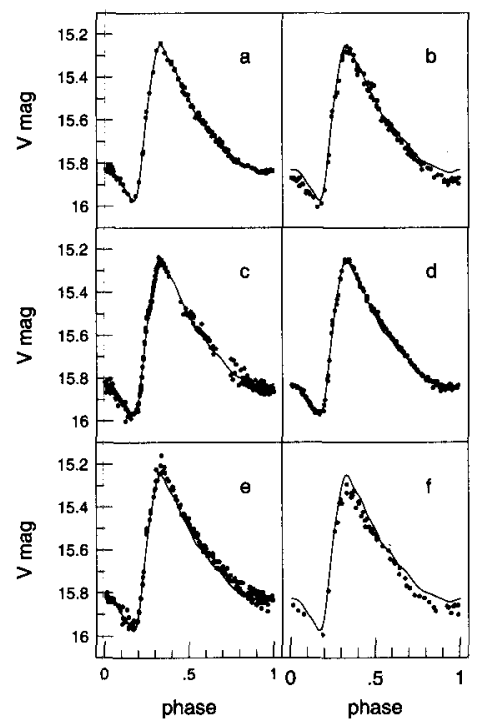

V 216

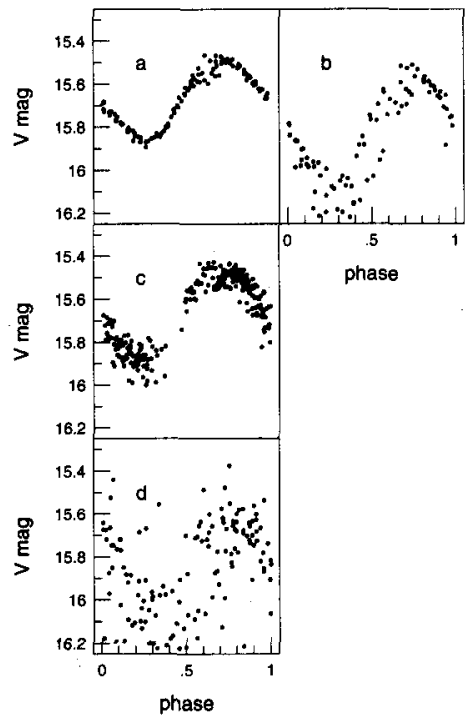

Figure 1. Different observations of an outer and an inner variables. V84: a) Konkoly 1-m, ISIS (BJ03); b) Konkoly 1-m, PSF (BJ03); c) Konkoly 60/90-cm Schmidt, ISIS (BJ03); d) La Palma 1-m, PSF (K98); e) Kitt Peak 90-cm, PSF (CC01); f) Loiano 1.5-m, PSF (C98). V216: a) Konkoly 1-m, ISIS (BJ03); b) Konkoly 1-m, PSF (BJ03); c) Konkoly 60/90-cm Schmidt, ISIS (BJ03); d) Kitt Peak 90-cm, PSF (CC01).

thus it is accurate for all those stars which are not seriously merged just in these best frames. Accordingly, the internal accuracy of the magnitudes in the reference frame defines the accuracy of the mean magnitudes of the variables. For those variables used in the present study the mean magnitudes are supposed to be correct within 0.02 in the BJ03 photometry. Supposedly the mean magnitudes in the (CC01) dataset are less accurate, those data were shifted, when necessary, in magnitude to the BJ03 data to match the light curves. No systematic differences were found between the mean magnitudes derived from the BJ03 and K98 data of the 26 stars in common. The mean difference between the mean magnitudes is $0.005 \pm 0.010$. The same data from the comparison of the BJ03 light curves with CC01 and C98 observations are $0^{\mathrm{m}} 019 \pm 0^{\mathrm{m}} 03$ for 82 stars, and $-00^{\mathrm{m}} 008 \pm 0.035$ for 28 stars, respectively. These results show that the BJ03 and K98 measurements are in very good agreement, while the CC01 light curves seem to be systematically brighter by $00^{\mathrm{m}} 02$ (this tendency was already mentioned in CC01), and the largest differences are in the $\mathrm{C} 98$ data, without any systematics. 

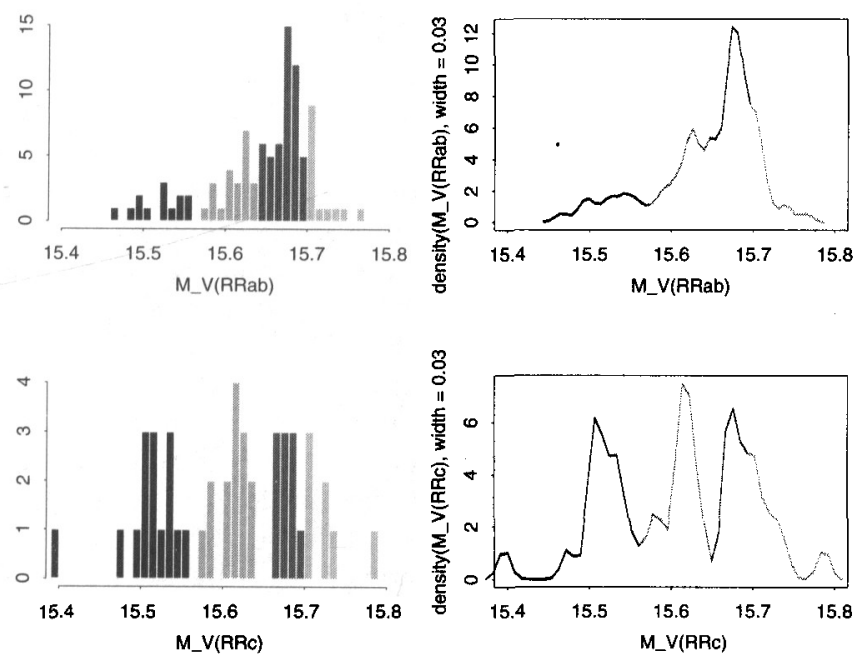

Figure 2. Histograms and density functions of the $\langle V\rangle$ magnitude distributions of RRab and RRc variables.

\section{Results}

Fig. 2. shows the $\langle V\rangle$ magnitude distributions of the variables. The mean magnitudes of both the RRab and RRc stars do not show a smooth, normal distribution but span $\sim 0^{\mathrm{m}} 3$ which can be divided into four different parts. For RRab stars the most populous group is at $15^{\mathrm{m}} 67$ (41 stars), there is a $0 .^{\mathrm{m}} 05$ brighter sample (22 stars), and the brightest stars show a flat magnitude distribution within the $15^{\mathrm{m}} 46-15^{\mathrm{m}} \cdot 56$ range (14 stars). Remembering that we assume the mean magnitudes to be correct within \pm 0 m 02 , observational inaccuracies cannot account for the existence of either the brightest stars or for the variables below $15 \mathrm{~m} .7$. The separation of the four groups is even more conspicuous for RRc stars. Among the overtone variables most of the stars belong to one of the brighter groups. Investigating the properties of the variables belonging to these four groups the following conclusions can be drawn.

1) The four groups at different mean brightness can be undoubtedly identified with different stages of the horizontal branch stellar evolution (Jurcsik et al. 2003). According to the comparison with Dorman's (1992) evolutionary models, stars fainter than $\langle V\rangle=15^{\mathrm{m}} 7$ are close to the ZAHB, variables between 15.65 and 15.7 are evolving blueward, at around 15.62 they are at the bluest part of their HB evolutionary tracks, while the brighter variables are already in the late, redward phase of their HB evolution.

2) The Fourier parameters of the light curves of RRab stars at different mean magnitudes follow different sequences along the periods similarly as predicted by hydrodynamical models of same mass, luminosity, composition models at different temperatures (Dorfi \& Feuchtinger 1999). The differences between these sequences are the most evident for the brighter stars and are marginal, if any, for the faintest sample. 
3) The mean periods of the RRab stars and the percentages of the RRc variables in the two fainter samples and for the brightest stars $(0.55 \mathrm{~d}$, and $22 \% ; 0.67 \mathrm{~d}$, and $56 \%$, respectively) correspond to Oosterhoff I and Oosterhoff II properties. This fact reveals that the Oosterhoff dichotomy can be present in a single cluster and that it can be interpreted in the context of horizontal branch stellar evolution as it has already been suggested by Clement \& Shelton (1999).

4) The period shifts observed between the different magnitude samples agree excellently with the period shift estimates arising from the detected inclination of the instability strip of RRab stars (Jurcsik 1998).

5) Rood and Crocker (1979) showed that the period distribution of synthetic HB simulations cannot reproduce the observed peaky period distribution of the M3 variables. This result was recently discussed and confirmed by Catelan (these proceedings). Adding together the period distributions of the single mode RRab stars at different brightness a peak in the period distribution can be detected in the $0.50-0.52-\mathrm{d}$ period interval. However, it is by far not so pronounced as is shown by Catelan (these proceedings) and in other similar works (e.g., CC01).The main difference between our period distribution and other results is that in our sample periods only of the single-mode RRab stars and some of those with small modulation amplitude are regarded. Therefore, we conclude that the concentration of the periods in the $0.5-0.55$-d period interval can be connected with the large number of Blazhko stars in M3, which phenomenon most probably does not have an evolutionary origin.

Acknowledgments. This work was supported by OTKA grant T-043504.

\section{References}

Alard, C. 2000, A\&AS, 144, 363

Benkő, J., Jurcsik, J., Bakos, G. et al. 2003, in preparation (BJ03)

Carretta, E. Cacciari, C., Ferraro, F.R., et al. 1998, MNRAS, 298, 1005 (C98)

Clement, C., Shelton, I. 1999, ApJ, 515, L85

Corwin, T. M., Carney, B. W. 2001, AJ, 122, 3183 (CC01)

Dorfi, E. A., Feuchtinger, M. 1999, A\&A, 348, 815

Dorman, B. 1992, ApJS, 81, 221

Jurcsik, J. 1998, A\&A, 333, 571

Jurcsik, J., Benkő, J., Bakos, G. Szeidl, B., Szabó R. 2003, ApJ, 547, L49

Kaluzny, J., Hilditch, R. W., Clement, C., Rucinski, S. M. 1998, MNRAS, 296, 347 (K98)

Olech, A., Wozniak, P., Alard, C., Kaluzny, J., Thompson, I. 1999, MNRAS, 310,759

Rood, R. T., Crocker, D. A. 1989, IAU Coll. 111, p. 218 


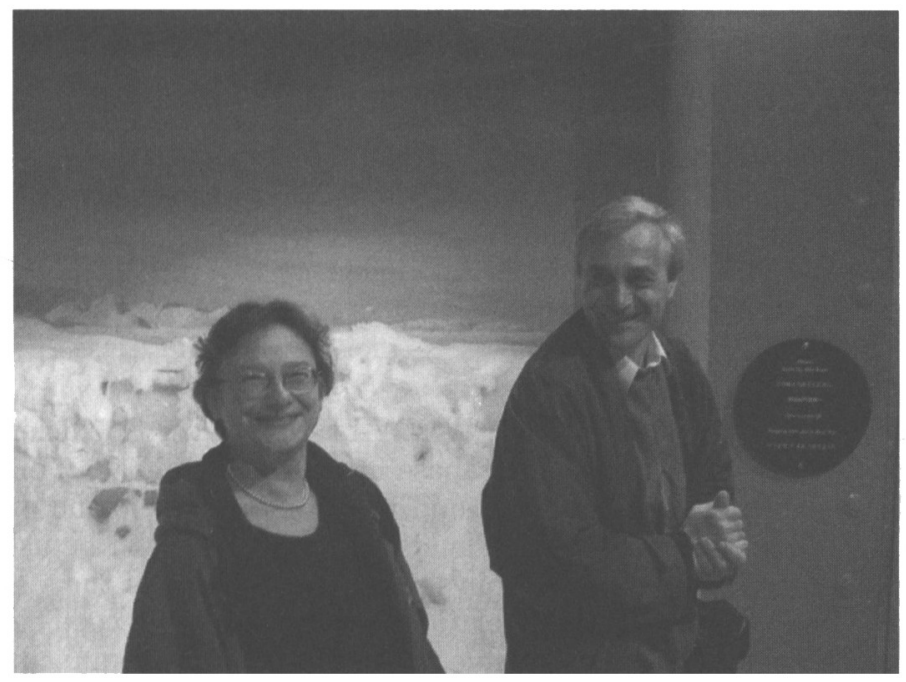

Johanna Jurcsik and Sandor Rostas

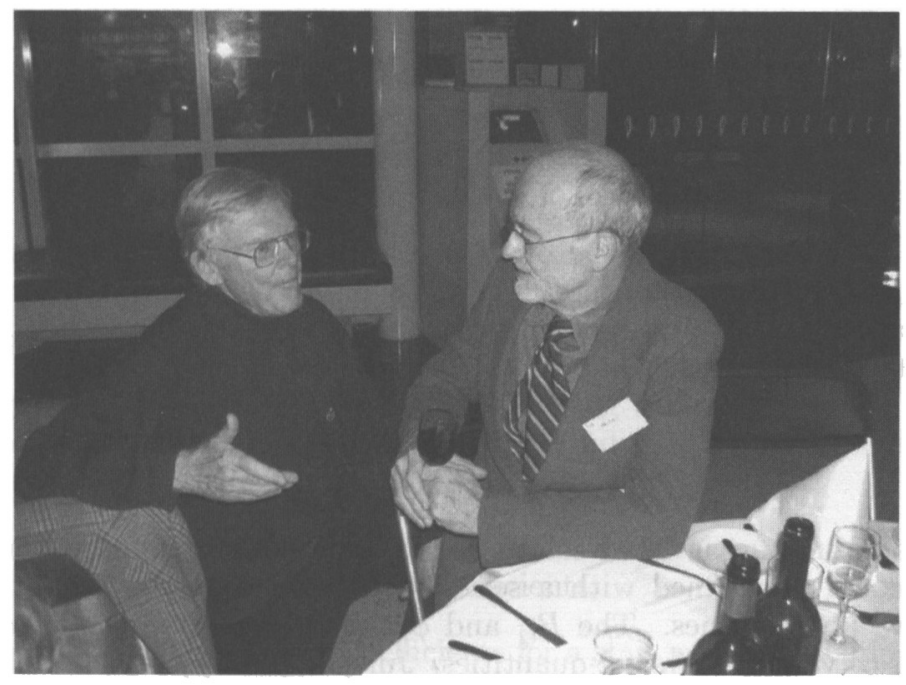

Bill Liller and Michael Snowden 